\title{
Improvement of Resolution in Nano-fabrication of GaN by Wet- chemical-assisted Femtosencond Laser Ablation
}

\author{
Seisuke Nakashima $^{1}$, Koji Sugioka ${ }^{1}$, and Katsumi Midorikawa ${ }^{1}$ \\ ${ }^{1}$ RIKEN - Advanced Science Institute, 2-1 Hirosawa, Wako, Saitama 351-0198, Japan \\ E-mail: seisuke@riken.jp
}

\begin{abstract}
Nano-fabrication technique for wide band-gap semiconductor gallium nitride (GaN) using a second harmonic of near infrared femtosecond ( $\mathrm{fs}$ ) laser $(\lambda=387 \mathrm{~nm}, 150 \mathrm{fs})$ has been investigated. We have carried out a wet-chemical-assisted fs laser ablation method, in which the laser beam is focused onto a single-crystal $\mathrm{GaN}$ substrate immersed in a hydrochloric $(\mathrm{HCl})$ or a phosphoric $\left(\mathrm{H}_{3} \mathrm{PO}_{4}\right)$ acid solution. The highest quality and best uniformity with less residual debris were obtained by using a concentrated $\mathrm{HCl}$ solution and thus nanoscale craters as small as $320 \mathrm{~nm}$ at full width at half maximum were formed by this technique. The second harmonic used in this work enhanced the fabrication resolution compared with the case of a fundamental beam of fs laser used in the previous report. We have also demonstrated the formation of two-dimensionally (2D) periodic nanostructures on surface of a $\mathrm{GaN}$ substrate using the second harmonic single fs-laser pulse of $110 \mathrm{~nJ}$. Ablation craters show a symmetrical-round shape with a diameter as small as 670 $\mathrm{nm}$ and with a depth of $60 \mathrm{~nm}$. Such structures are applicable to fabrication of 2D photonic crystals which improve the light extraction efficiency for blue LEDs in the near future.
\end{abstract}

DOI: $10.2961 / \mathrm{jlmn} .2010 .01 .0005$

Keywords: Femtosecond laser, ablation, Gallium nitride, nano-fabrication

\section{Introduction}

Recently, GaN-based light-emitting diodes (LEDs) are being widely used for many applications everywhere such as liquid crystal display back lightings, full color displays, traffic lights, and other general lighting purposes. Further development for the existing devices and more advanced applications demand higher light output with lower power consumption. However, only a very limited fraction of the photons generated inside a GaN LED can go out of the devices because of total internal reflection at an interface between $\mathrm{GaN}(\mathrm{n}=2.5)$ and air $(\mathrm{n}=1)$. The extraction efficiency for the $\mathrm{GaN}$ /air single interface is less than $10 \%$ in spite of the fact that an internal quantum efficiency of up to $90 \%$ is realized. Since typical GaN-based LED structures are grown on a sapphire substrate, photons are not only reflected back but also be guided laterally through the GaN waveguide between air and sapphire, resulting in either being reabsorbed by quantum well active layer or radiating in less useful directions. In order to circumvent this problem, integration of two-dimensional (2D) photonic crystal patterns with air holes on the device surface is proposed [13]. In such structures, the total internal reflection is suppressed, and in-plane lateral guide modes of light are forbidden, as a result, a vertical waveguiding effect induces photon emission in the off-plane direction, which leads to an enhancement of the extraction efficiency.

To fabricate 2D photonic crystals on devices, electron beam lithography followed by plasma etching processes are utilized as existing technologies $[4,5]$. It has been reported that they can form a periodic structure with nano-sized air deep holes as small as about $150 \mathrm{~nm}$. In this method, however high cost of production is unavoidable, and low throughput due to complicated processes and small fabrication area make the mass production difficult. Some other approaches to fabricate $2 \mathrm{D}$ photonic crystals, but they are not breakthrough technology at present [6-9].

Meanwhile, in our past work, we investigated the possibility of micro/nanofabrication of GaN using femtosecond laser ablation. In our previous report, nano-sized crater can be successfully formed by using wet-chemicalassisted fs laser ablation method $[10,11]$. Since the fs laser ablation is direct and simple process, we propose that this laser ablation is applicable to fabrication method of $2 \mathrm{D}$ photonic crystals with high efficiency and high throughput of production. In this paper, preliminary experiments for practical application are carried out. Compared to the existing methods, fabrication resolution performed in the previous work was lower. Therefore, the second harmonic of near infrared fs laser is utilized for fabrication. Then, we try to fabricate simple periodic structures on surfaces of GaN substrates.

\section{Experimental}

The material used in this study was (0001)-orientated single-crystal GaN substrates (Kyma Technologies, Inc.). These substrates, which were $470 \mu \mathrm{m}$ thick, exhibit n-type conduction. We utilized a fs laser (Clark-MXR, CPA2001) that emits pulses with pulse widths of $150 \mathrm{fs}$ [full width at half maximum (FWHM)], a wavelength of $387 \mathrm{~nm}$ (SHG) produced by a $\mathrm{BBO}$ crystal, and a repetition rate of $1 \mathrm{kHz}$. The laser beam was shaped by an aperture to a diameter of $3 \mathrm{~mm}$ and was then focused onto the substrate surface by a $\times 39$ microscope objective lens with a numerical aperture (NA) of 0.5 and with a working distance of 2.0 $\mathrm{mm}$. The GaN substrates were translated using a PCcontrolled xyz stage with a resolution of $0.5 \mu \mathrm{m}$. We performed wet-chemical-assisted ablation, in which the laser beam was focused directly onto a substrate immersed in a 
hydrochloric acid $(\mathrm{HCl})$ or phosphoric acid $\left(\mathrm{H}_{3} \mathrm{PO}_{4}\right)$ solution. The thickness of acid solution on the surface of substrates was kept constant to be $630 \mu \mathrm{m}$. Both of the solutions are almost completely transparent at the wavelength of $775 \mathrm{~nm}$ as well as $387 \mathrm{~nm}$. In our previous work, we reported that this method was better adapted to higher quality and higher throughput fabrication than two-step processing method, i. e., fs laser ablation followed by $\mathrm{HCl}$ treatment [11]. The feature and size of the ablation crater formed by a single fs-laser pulse were investigated by atomic force microscopy (AFM). Cross-sectional profiles were also derived from the AFM data, leading to measurements of depths and FWHM diameters for each crater.

\section{Results and discussions}

Shown in Figure 1(a) is AFM images for craters formed by the wet-chemical-assisted laser ablation using a $3.5 \%$ $\mathrm{HCl}$ acid solution. Each crater was fabricated by a single shot of laser beam. In our system, an electro-mechanical shutter was equipped to select the desired number of pulses from a pulse-train with a repetition rate of $1 \mathrm{kHz}$. Since the shortest shutter opening time was limited to $0.01 \mathrm{~s}$, the minimum number of laser pulses that can be applied to the same spot is 10 . For single pulse irradiation at each spot, the substrate was translated on the xyz stage with a speed of $5000 \mu \mathrm{m} / \mathrm{s}$. Hence, a series of craters $5 \mu \mathrm{m}$ apart from each other were formed by the same irradiation condition. The pulse energies for fabrication are $60,70,90$, and 400 $\mathrm{nJ}$, respectively. At the pulse energy of $60 \mathrm{~nJ}$, the averaged ablation crater is as small as $700 \mathrm{~nm}$, while no crater was formed by irradiation at the energy lower than $60 \mathrm{~nJ}$. As increasing in the pulse energy, the ablation crater becomes larger, and the shape changes from a symmetrical round to an ellipse and at higher pulse energy the ablation crater is
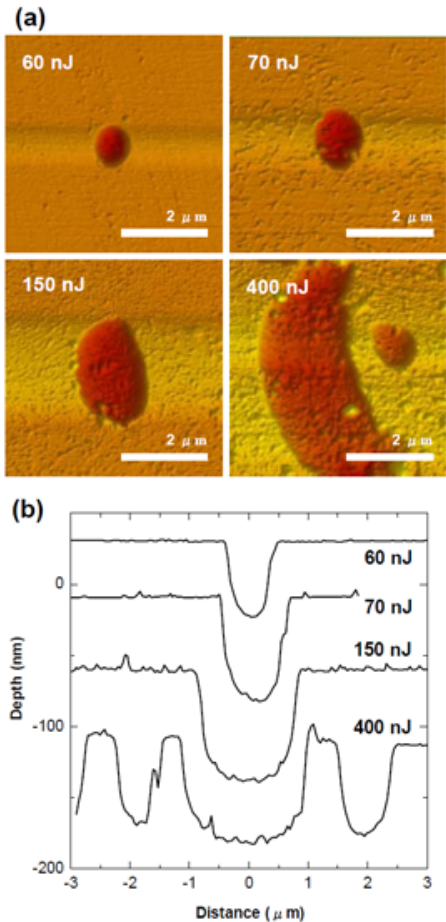

Figure 1 (a) AFM images and (b) cross-section profiles of ablation craters formed by a wet-chemical-assisted fs laser ablation in 3.5\% $\mathrm{HCl}$ acid solution. far from the circle. The distortion in the shape of crater is presumably ascribed to the interference of bubbles generated at the neighboring ablation crater by the previous pulse. In fact, such distortion was not observed in the case of ablation in air. Figure 1(b) shows cross-sectional profiles, which indicate that the edges and surfaces of some ablation craters are unclear and rough. The high roughness not only at crater's surface but also at the substrate's surface is probably attributable to residual debris.

In order to check an effect on the concentration, a concentrated $\mathrm{HCl}$ solution (35\%) is used. As shown in Fig. 2(a), high quality ablation craters with better round shape are obtained at the pulse energy lower than $70 \mathrm{~nJ}$. Unlike the case of using a diluted $\mathrm{HCl}$ solution, residual debris is almost completely removed by the concentrated acid solution. In Fig. 2(b), cross-sectional profiles also illustrate the smoothness of craters' surface. An ablation crater as small as $320 \mathrm{~nm}$ is successfully fabricated at the pulse energy of $65 \mathrm{~nJ}$. This fabrication resolution is higher than the case of using fundamental light of near-infrared fs laser [11].

We also tried to use $30 \% \mathrm{H}_{3} \mathrm{PO}_{4}$ for finding out optimum acid solution for this process. Figure 3 exhibits the results of AFM measurement; (a) height images and (b) cross-sectional profiles for craters, respectively. At the pulse energy of $60 \mathrm{~nJ}$, the nano-sized craters as small as $400 \mathrm{~nm}$ are obtained, but the formed shape looks somewhat distorted. As increasing in the pulse energy, the ablation crater becomes larger, and the shape changes from round to irregular similarly to the case of $\mathrm{HCl}$ acid. At the pulse energy higher than $400 \mathrm{~nJ}$, the craters' surface conditions become much worse. Considering all data, the wetchemical-assisted ablation using concentrated $\mathrm{HCl}$ acid solution is suited for fabrication of smooth craters with higher resolution and better quality.

Figure 4 shows a variation in crater depth as a function
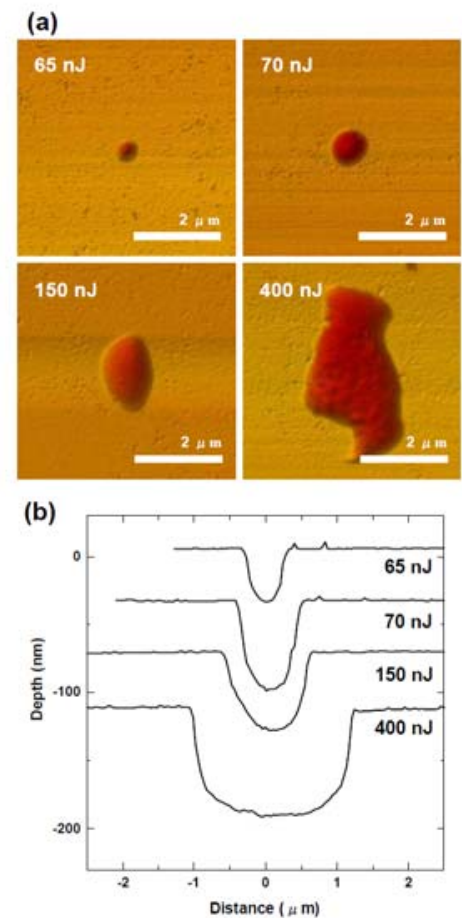

Figure 2 (a) AFM images and (b) cross-section profiles of ablation craters formed by a wet-chemical-assisted fs laser ablation in $35 \%$ $\mathrm{HCl}$ acid solution. 

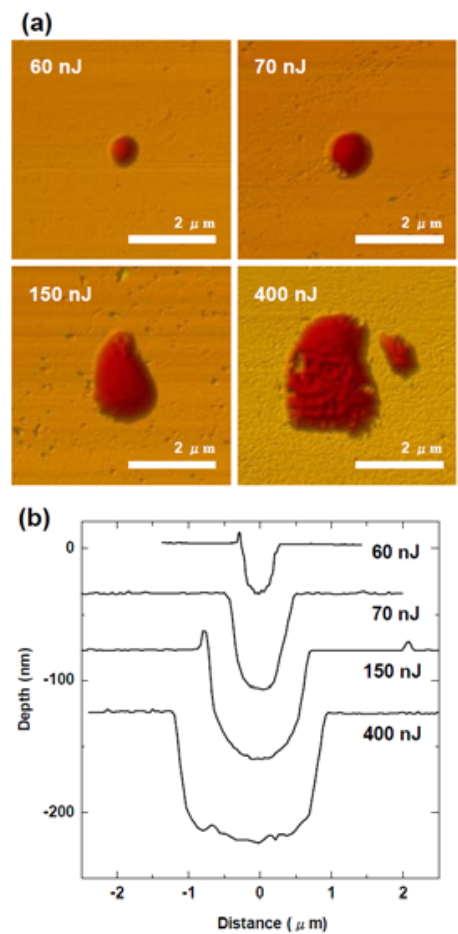

Figure 3 (a) AFM images and (b) cross-section profiles of ablation craters formed by a wet-chemical-assisted fs laser ablation in $30 \%$ phosphoric acid solution.

of pulse energy for concentrated $\mathrm{HCl}$ acid. The depth of ablation craters tends to be saturated at $80 \mathrm{~nm}$. Band gap of the $\mathrm{GaN}$ is $3.4 \mathrm{eV}$, which is so large that a single photon of SHG light can not excite an electron from the valence band to the conduction band. Therefore, it is speculated that the ablation reaction takes place by two-photon absorption. In the two-photon absorption, generally, absorption is related to 2 nd power of the intensity as;

$$
\frac{d I}{d x}=-\alpha \cdot I^{2}
$$

where $\alpha$ is the nonlinear absorption coefficient and $x$ is the distance from the surface. When an ablation crater with a depth of $X \mathrm{~nm}$ was created by an irradiation of a GaN surface with focused fs laser, the propagated laser intensity at the depth of $X$ corresponds to the threshold intensity of ablation, $I_{t h}$. Integrating Eq. (1) from the surface where laser intensity is $I_{0}$ to the depth $X$ where laser intensity is $I_{t h}$, the depth of ablation craters is expressed by

$$
X=\frac{1}{\alpha}\left(\frac{1}{I_{t h}}-\frac{1}{I_{0}}\right)
$$

From a fit of this equation to the experimental depth data (solid line in Figure 4), $I_{t h}$ for ablation of $\mathrm{GaN}$ is estimated as $34.8 \mathrm{~nJ}$. By the way, a beam profile of fs laser is expressed using Gaussian function as;

$$
I(r)=I_{0} \exp \left(-\frac{2 r^{2}}{\omega_{0}^{2}}\right),
$$

where $r$ denotes a distance from the center of beam and $I(r)$ represents the intensity at the position of $r$. Gaussian beam radius $\omega_{0}$ was estimated as $0.64 \mu \mathrm{m}$ by an equation of

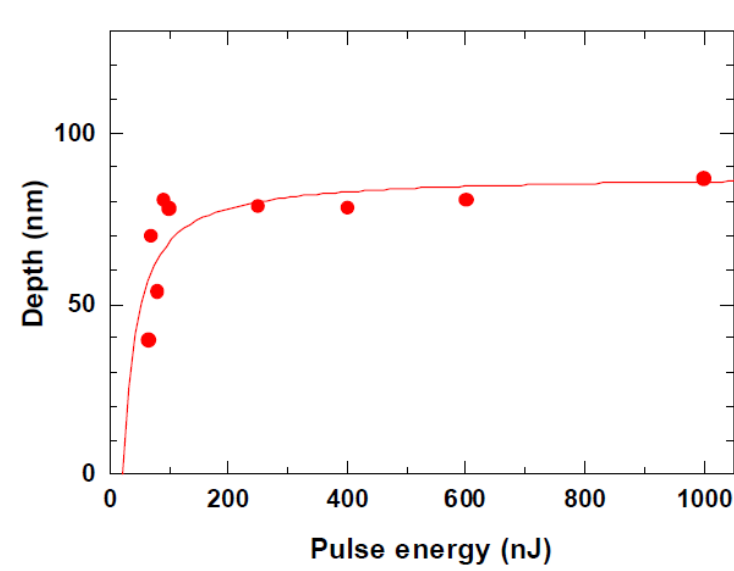

Figure 4 Variation in crater depth as a function of pulse energy for concentrated $\mathrm{HCl}$ acid. Solid line is a fit with the Equation 1 based on optical absorption due to two-photon absorption.

$\omega_{0}=0.64 \cdot \lambda / N A \cdot M^{2}$. Here, $M^{2}$ for our fs-laser system is $1.3, \mathrm{NA}=0.5$ and $1=387 \mathrm{~nm}$. Since the incident laser intensity $I_{0}$ is converted into the total pulse energy $P(\infty)$ by an equation of $I_{0}=2 \cdot P(\infty) / \pi \omega_{0}^{2}, I(r)$ can be expressed using $P(\infty)$ as;

$$
I(r)=\frac{2 P(\infty)}{\pi \omega_{0}{ }^{2}} \exp \left(-\frac{2 r^{2}}{\omega_{0}{ }^{2}}\right)
$$

Since only the region where the laser intensity exceeds $I_{t h}$ should be ablated, a theoretical diameter of ablation crater, $D\left(=2 r\left(I_{t h}\right)\right)$ associated with $I_{t h}$ can be calculated by

$$
D=2 r_{t h}=\omega_{0} \sqrt{\ln \left(\frac{2 P}{\pi \omega_{0}^{2} I_{t h}}\right)}
$$

In Fig. 5, a calculated diameter of ablation craters is plotted for various laser pulse energies. The experimental data of diameter of craters for the concentrated $\mathrm{HCl}$ are also shown using red circles. In the region of energy lower

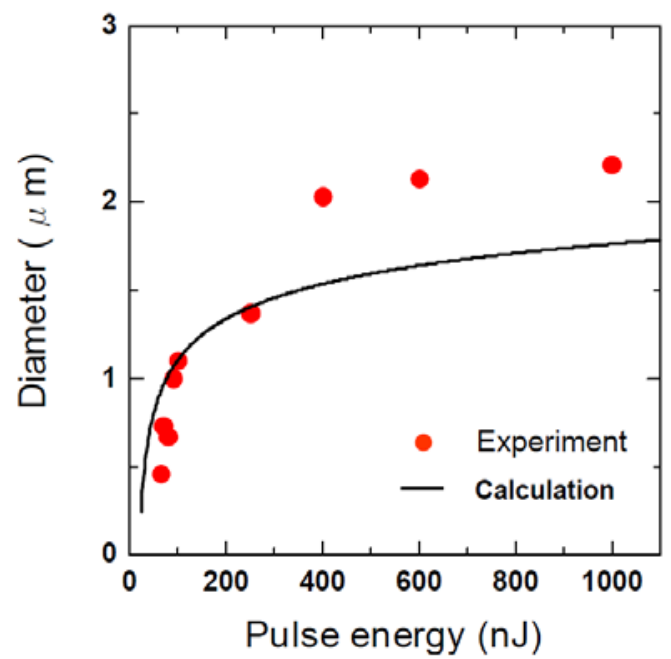

Figure 5 Calculated diameter of ablation craters as a function of pulse energy for concentrated $\mathrm{HCl}$ solution. Red circles represent experimental data. 
than $250 \mathrm{~nJ}$, the calculation well agrees with the experimental values. By contrast, in the region of energy higher than $400 \mathrm{~nJ}$, calculated diameters are underestimated compared with experimental data. At present, the reason of this discrepancy is not clear, but we speculate that this discrepancy is probably due to effect of thermal diffusion or to the distortion of beam shape interfered by bubbles as discussed before (see Fig. 2). In this work, a high resolution $(\sim 320$ $\mathrm{nm})$ fabrication has been achieved using the wet-chemicalassisted fs laser ablation based on multiphoton absorption of SHG light at the lowest pulse energy of $65 \mathrm{~nJ}$.

We have tried to fabricate preliminary 2D periodic structures on $\mathrm{GaN}$ substrates. A schematic diagram for fabrication of periodic structures is shown in Figure 6(a). A 2D structure is simply composed of arrangement of ablation craters formed by single pulse irradiation with fs laser. In the $\mathrm{x}$ direction, the focused laser beam is scanned with a scanning speed $5000 \mu \mathrm{m} / \mathrm{s}$, leading to an equally-arranged ablation craters on a line. After moving the stage in the $y$ direction with a pitch of $5 \mu \mathrm{m}$, the next line is scanned repeatedly. The distance between craters, in other word, periodicity is adjustable by the change of scanning speed. Figure 6(b) shows an AFM image for a simple 2D structure, in which ablation holes were formed at pulse energy of 110 $\mathrm{nJ}$ with a periodicity of $5 \mu \mathrm{m}$. The crater diameter and depth are $670 \mathrm{~nm}$ and $60 \mathrm{~nm}$, respectively. Although uniform nano-sized ablation craters are periodically distributed in a broad area, this structure does not still work as a 2D photonic crystal for blue LED because of the unsatisfactory resolution and periodicity as well as the shallow crater depth. It is necessary to obtain smaller crater and to improve accuracy in periodicity. For higher resolution and smaller periodicity, the stability of stage transferring should be improved. To increase the crater depth, increase of pulse numbers irradiated to the same spot is required, for which a pulse control by changing the repetition rate would be effective.

\section{Conclusions}

Fabrication resolution in processing of GaN surface by wet-chemical-assisted ablation in a concentrated $\mathrm{HCl}$ solution is improved by using the second-harmonic of nearinfrared femtosecond laser. Successfully formed nano craters are as small as $320 \mathrm{~nm}$ in diameter. Ablation threshold peak intensity is estimated by assuming that the laser ablation is due to two-photon absorption, and we calculated the theoretical diameter of ablation craters. In lower energy, the calculated diameters are very close to the experimental values, while the diameter in the region of higher energy is underestimated. We also demonstrated the fabrication of 2D structure on a GaN substrate, in which uniform ablation craters as small as $670 \mathrm{~nm}$ are arranged, indicating the possibility for a fabrication of $2 \mathrm{D}$ photonic crystal structures on the surface of LED based on GaN.

(Received: August 14, 2009, Accepted: December 21, 2009) (a)

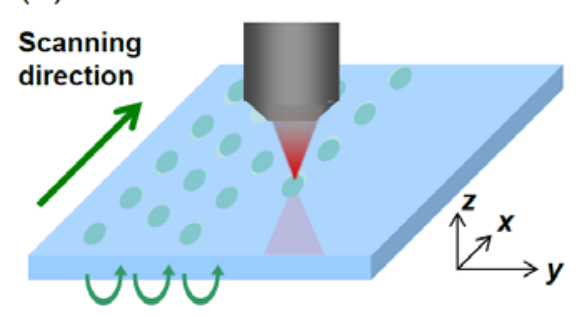

(b)

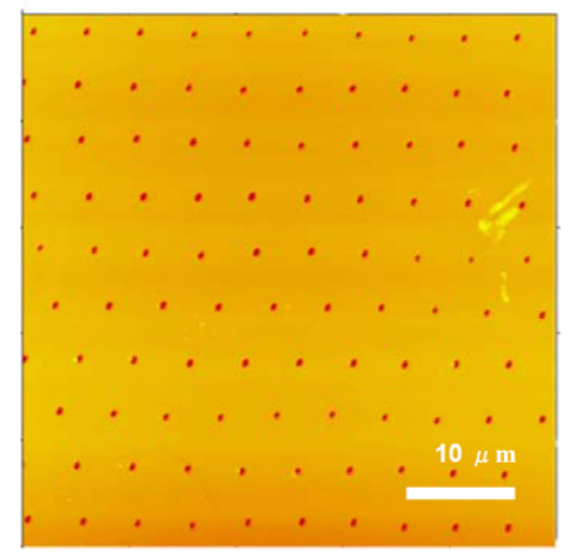

Figure 6 (a) Schematic diagram of fabrication of 2D periodic structures. (b) AFM image for a simple 2D structure, in which ablation holes were formed at pulse energy of $110 \mathrm{~nJ}$ with a periodicity of 5 um.

\section{References}

[1] S. Fan, P. R. Villeneuve, J. D. Joannopoulos, and E. F. Schubert, Phys. Rev. Lett., 78, (1997) 3294. (Journals)

[2] E. Yablonobitch, T. J. Gmitter, and R. Bhat, Phys. Rev. Lett., 61, (1988) 2546. (Journals)

[3] T. N. Oder, H. S. Kim, J. Y. Lin, and H. X. Jiang, Appl. Phys. Lett., 84, (2004) 466. (Journals)

[4] C. Meier, K. Hennessy, E. D. Haberer, R. Sharma, Y.S. Choi, K. McGroddy, S. Keller, S. P. DenBaars, S. Nakamura, and E. L. Hu, Appl. Phys. Lett., 88, (2006) 031111. (Journals)

[5] K. Kim, J. Choi, S. C. Jeon, J. S. Kim, and H. M. Lee, Appl. Phys. Lett., 90, (2007) 181115. (Journals)

[6] Z. S. Zhang, B. Zhang, J. Xu, K. Xu. Z. J. Yang, Z. X. Qin, T. J. Yu, and D. P. Yu, Appl. Phys. Lett., 88, (2006) 171103. (Journals)

[7] M.-K. Kwon, J.-Y. Kim, I.-K. Park, K. S. Kim, G.-Y. Jung, S.-J. Park, J. W. Kim, and Y. C. Kim, Appl. Phys. Lett. 92, (2008) 251110. (Journals)

[8] W. N. Ng, C. H. Leung, P. T. Lai, and H. W. Choi, Nanotechnology 19, (2008) 255302. (Journals)

[9] T. A. Truong, L.M. Campos, E. Matioli, I. Meinel, C. J. Hawker, and P. M. Pertroff, Appl. Phys. Lett., 94, (2009) 023101. (Journals)

[10] S. Nakashima, K. Sugioka, K. Midorikawa, J. Laser Micro/Nanoeng., 4, (2009) 75. (Journals)

[11] S. Nakashima, K. Sugioka, K. Midorikawa, Appl. Surf. Sci. in press. 